\title{
Diazoxide protects against doxorubicin-induced cardiotoxicity in the rat
}

\author{
Lisa Drange Hole ${ }^{1 *}$, Terje Hjalmar Larsen ${ }^{2,3}$, Kjell Ove Fossan ${ }^{1}$, Fredrik Limé ${ }^{1}$ and Jan Schjøtt ${ }^{1,4}$
}

\begin{abstract}
Aim: Chemotherapy with doxorubicin is limited by cardiotoxicity. Free radical generation and mitochondrial dysfunction are thought to contribute to doxorubicin-induced cardiac failure. In this study we wanted to investigate if opening of mitochondrial $\mathrm{K}_{\mathrm{ATP}}$-channels by diazoxide is protective against doxorubicin cardiotoxicity, and if 5-hydroxydecanoate (5-HD), a selective mitochondrial $\mathrm{K}_{\text {ATP }}$-channel antagonist, abolished any protection by this intervention.

Methods: Wistar rats were divided into 7 groups $(n=6)$ and followed for 10 days with 5 intervention groups including the following treatments: (1) Diazoxide and doxorubicin, (2) diazoxide and 5-hydroxydecanoate (5-HD), (3) 5-HD and doxorubicin, (4) diazoxide and saline and (5) 5-HD and saline. On day 1,3,5 and 7 the animals received intraperitoneal (i.p.) injections with $10 \mathrm{mg} / \mathrm{kg}$ diazoxide and $/ \mathrm{or} 40 \mathrm{mg} / \mathrm{kg} 5-\mathrm{HD}, 30$ minutes before i.p. injections with $3.0 \mathrm{mg} / \mathrm{kg}$ doxorubicin. One control group received only saline injections and the other control group received saline 30 minutes prior to $3.0 \mathrm{mg} / \mathrm{kg}$ doxorubicin. On day 10 the hearts were excised and Langendorff-perfused. Cardiac function was assessed by an intraventricular balloon and biochemical effects by release of hydrogen peroxide $\left(\mathrm{H}_{2} \mathrm{O}_{2}\right)$ and troponin- $\mathrm{T}$ (TnT) in effluate from the isolated hearts, and by myocardial content of doxorubicin.

Results: Doxorubicin treatment produced a significant loss in left ventricular developed pressure (LVDP) $(p<0.05)$ and an increase in both $\mathrm{H}_{2} \mathrm{O}_{2}$ and TnT release in effluate $(p<0.05)$. Diazoxide significantly attenuated the decrease in LVDP $(p<0.05)$ and abolished the increased release of $\mathrm{H}_{2} \mathrm{O}_{2}$ and $\operatorname{TnT}(p<0.05)$. 5- HD abolished the effects of pretreatment with diazoxide, and these effects were not associated with reduced myocardial accumulation of doxorubicin.
\end{abstract}

Conclusions: Pretreatment with diazoxide attenuates doxorubicin-induced cardiac dysfunction in the rat, measured by physiological indices and TnT and $\mathrm{H}_{2} \mathrm{O}_{2}$ in effluate from isolated hearts. The effect could be mediated by opening of

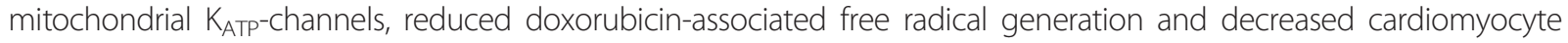
damage. Diazoxide represents a promising protective intervention against doxorubicin-induced acute cardiotoxicity.

Keywords: Doxorubicin, Troponin T, Hydrogenperoxide, Doxorubicinol, Heart, Rat, 5-hydroxydecanoate, Cardiotoxicity, Ex vivo, Diazoxide

\section{Background}

Doxorubicin is a widely used chemotherapy drug, but its application is associated with cardiotoxicity. Free radical generation and mitochondrial dysfunction are thought to contribute to doxorubicin-induced cardiac failure [1,2]. Dose reduction protocols have been proposed to avoid the risk of delayed cardiotoxicity, but this might be at the expense of the anticancer effect [3]. In addition, impaired calcium handling and cellular damage mediated

\footnotetext{
* Correspondence: lisa.drange.hole@helse-bergen.no

${ }^{1}$ Section of Clinical Pharmacology, Laboratory of Clinical Biochemistry,

Haukeland University Hospital, 5021 Bergen, Norway

Full list of author information is available at the end of the article
}

by reactive oxygen species, have been proposed as toxic mechanisms to explain both acute and delayed cardiotoxicity of anthracyclines [4-6].

Diazoxide has been in clinical use since the early 1960's to treat severe non-malignant and malignant hypertension in hospitalized adults and acute severe hypertension in hospitalized children. Diazoxide is also used to treat hypoglycaemia. The mechanisms of diazoxide's clinical action relate predominantly to the opening of pancreatic and smooth muscle $\mathrm{K}_{\mathrm{ATP}}$-channels [7].

Mitochondria are the major effectors of cardioprotection by mechanisms that open the mitochondrial $\mathrm{K}_{\mathrm{ATP}}$-channel, including ischemic and pharmacological 
preconditioning [8]. Pharmacological preconditioning, mimicking ischemic preconditioning, is suggested as an intervention to reduce doxorubicin cardiotoxicity [9]. Morphine has been demonstrated to mimic preconditioning in cardiomyocytes [10,11]. We have previously observed increased mortality in rats pretreated in vivo with morphine before doxorubicin (32). In a follow-up study we pretreated rats with morphine, isolated the hearts and exposed them to doxorubicin in a Langendorff perfusion system (unpublished data). We found that pretreatment with morphine in vivo was associated with a cardiodepressive effect in isolated hearts before doxorubicin exposure. After exposure to doxorubicin ex vivo, isolated hearts from rats pretreated with morphine were associated with increased release of hydrogen peroxide $\left(\mathrm{H}_{2} \mathrm{O}_{2}\right)$, increased release of troponin- $\mathrm{T}$ (TnT), increased myocardial contracture and increased myocardial content of doxorubicin. These findings were in contrary to results from a comparable study [9] which found that morphine was protective against doxorubicin cardiotoxicity. Based on these opposing results we wanted to investigate further, whether pharmacological preconditioning could reduce doxorubicin cardiotoxicity.

Direct stimulation of myocardial $\delta_{1}$-opioid receptors leads to opening of mitochondrial $\mathrm{K}_{\mathrm{ATP}}$-channels and $\mathrm{a}$ resultant increase in intracellular free radical signals in vitro [10]. Recently, nicorandil, a mitochondrial $\mathrm{K}_{\mathrm{ATP}^{-}}$ channel opener, has successfully been administered to counteract the toxic effects of doxorubicin [12,13]. In light of our previous results, we now wanted to bypass the opioid receptors in this experiment, and study how direct opening of mitochondrial $\mathrm{K}_{\mathrm{ATP}}$-channels interacts with the cardiotoxic mechanisms of anthracyclines. We used diazoxide, a selective mitochondrial $\mathrm{K}_{\mathrm{ATP}}$-channel agonist, known to have protective properties against cardiac ischemia $[7,14]$. We also used 5-hydroxydecanoate (5-HD), a selective mitochondrial $\mathrm{K}_{\mathrm{ATP}}$-channel antagonist. 5-HD inhibits the increase in free radicals seen with $\delta_{1}$-opioid receptor activation, and abolishes cardioprotection afforded by ischemic preconditioning [7]. Our model combines drug treatment in vivo with the study of hearts ex vivo, yielding direct physiological information on systolic and diastolic cardiac function combined with biochemical indices like $\mathrm{H}_{2} \mathrm{O}_{2}$, TnT and measurement of myocardial content of doxorubicin.

\section{Methods}

\section{Materials}

Doxorubicin was purchased from Meda AS (Slemmestad, Norway), diazoxide, 5-hydroxydecanoate and pentobarbital from Haukeland Hospital Pharmacy (Bergen, Norway), heparin from Leo Pharma A/S (Oslo, Norway), and ingredients for the Krebs-Henseleit bicarbonate buffer from Merck KGaA (Darmstadt, Germany). This study conforms to the Guide for the Care and Use of Laboratory Animals published by the US National Institutes of Health (NIH Publication No. 85-23, revised 1996) and was approved by the Animal Care and User Committee in Norway with document number: ID 1766 FOTS, and certified institution number 066 Vivarium, University of Bergen.

\section{Animals}

Male Wistar rats weighing $200 \pm 20$ grams were purchased from Taconic (Ejby, Denmark). The animals were housed in grid-bottom metal wire cages in a room maintained at a 12 hour light/dark cycle at a temperature of $20-22^{\circ} \mathrm{C}$. They were acclimatised for two weeks, housed three per cage and allowed free access to food pellets (Pellets rodent, Special Diets Services, Essex, UK) and tap water until i.p. injection of diazoxide, 5-HD, saline or doxorubicin. The animals were separated in individual cages based on their respective treatment protocols.

\section{Langendorff perfusion model}

The perfusion medium was a modified, oxygenated $\left(95 \% \mathrm{O}_{2}\right.$ and $5 \% \mathrm{CO}_{2}$ ) Krebs-Henseleit bicarbonate buffer (KHBB) ( $\mathrm{pH} 7.4$ ) containing in $\mathrm{mM}: 118.5 \mathrm{NaCl}$, $25.0 \mathrm{NaHCO}_{3}, 1.2 \mathrm{MgSO}_{4}, 4.7 \mathrm{KCl}, 1.2 \mathrm{KH}_{2} \mathrm{PO}_{4}, 11.0$ D-glucose, and $1.25 \mathrm{CaCl}_{2}$. Hearts were excised after anaesthesia of the rats with an i.p. injection of pentobarbital $50 \mathrm{mg} / \mathrm{kg}(0.1 \mathrm{ml} / 100 \mathrm{~g}$ bodyweight $)$ and heparinised i.p. (0.1 ml $500 \mathrm{IU} / 100 \mathrm{~g}$ bodyweight). Anaesthesia was evaluated by the pedal-withdrawal reflex. The heart was rapidly excised and immediately placed in cold $\left(4^{\circ} \mathrm{C}\right)$ KHBB to temporarily stop its beating and preserve it from ischemic injury prior to perfusion. The heart was mounted on a steel cannula placed in the aorta and perfused retrogradely in a Langendorff system with the use of thermostated $\left(37^{\circ} \mathrm{C}\right)$ reservoirs (Lauda, LaudaKönigshofen, Germany), perfusion lines and heart chamber. Pressure regulated flow was performed at $100 \mathrm{cmH}_{2} \mathrm{O}$ (73 $\mathrm{mmHg})$, while volume regulated flow $(12.5 \mathrm{ml} / \mathrm{min})$ was performed by use of an Alitea peristaltic pump (Alitea, Stockholm, Sweden). A water-filled latex balloon was placed in the left ventricle and connected to a pressure transducer (Memscap AS, Skoppum, Norway) for the recording of left ventricular pressure (LVDP) and secondarily derived contractility indices. Left ventricular enddiastolic pressure (LVEDP) was adjusted between 4 and $8 \mathrm{mmHg}$. A second pressure transducer was connected to a side arm on the aortic cannula for the recording of aortic pressure (AoP), an index of coronary vascular resistance during volume-regulated perfusion. Pressure signals were amplified (Quadbridge, AD Instruments, London, UK) and recorded using a PowerLab data acquisition system (AD Instruments, East Sussex, UK). AoP, LVDP, LVEDP, left ventricular pressure first derivatives maximum $\left(\mathrm{dp} / \mathrm{dt}_{\max }\right)$ and minimum $\left(\mathrm{dp} / \mathrm{dt}_{\min }\right)$ were continuously 
displayed and recorded. Pacing (300 beats per minute by electric stimulation of $5 \mathrm{~V}$ amplitude of $3 \mathrm{~ms}$ duration) was obtained by placing one electrode on the right auricle and one on the steel cannula. Pacing was used to maintain a standard contractile response to the experimental drugs in the model not influenced by changes in heart rate and/or periods of arrhythmia. Coronary flow rate was measured by timed collection of the coronary perfusate that dripped from the heart. At the end of the perfusion protocol, hearts were removed from the Langendorff system and myocardial tissue from the left ventricle was dissected free and immediately frozen in liquid helium and stored at $-80^{\circ} \mathrm{C}$ until analysis of doxorubicin and doxorubicinol was performed, within 14 days of termination of the perfusion protocol. Effluent samples of $1 \mathrm{~mL}$ were collected in $1.5 \mathrm{~mL}$ polypropylene Eppendorf micro test tubes (Eppendorf Vertrieb, Wesseling-Berzdorf, Germany) from each heart, at the end of the perfusion protocol, and stored at $0^{\circ} \mathrm{C}$, until analysis for $\mathrm{TnT}$ within 4 days of termination of the perfusion protocol. Effluent samples of $1 \mathrm{~mL}$ were collected in Eppendorf tubes from each heart at the end of the perfusion protocol, placed in a thermostated $\left(37^{\circ} \mathrm{C}\right)$ Eppendorf rack heated by a Lauda reservoir (Lauda, Königshofen, Germany), and immediately analysed for $\mathrm{H}_{2} \mathrm{O}_{2}$. All experiments and analysis were carried out between 7 am and 7 pm.

\section{Experimental design}

DIADOX $(n=6)$ received pretreatment with an i.p. injection of $10 \mathrm{mg} / \mathrm{kg}$ diazoxide 30 minutes before an i.p. injection of $3 \mathrm{mg} / \mathrm{kg}$ doxorubicin.

DIA5HDDOX $(n=6)$ received pretreatment with an i.p. injection of $10 \mathrm{mg} / \mathrm{kg}$ diazoxide 30 minutes before an i.p. injection of $40 \mathrm{mg} / \mathrm{kg} 5-\mathrm{HD}, 30$ minutes before an i.p. injection of $3 \mathrm{mg} / \mathrm{kg}$ doxorubicin.

5HDDOX $(n=6)$ received pretreatment with an i.p. injection of $40 \mathrm{mg} / \mathrm{kg} 5$-HD 30 minutes before an i.p. injection of $3 \mathrm{mg} / \mathrm{kg}$ doxorubicin.

DIASAL $(n=6)$ received pretreatment with an i.p. injection of $10 \mathrm{mg} / \mathrm{kg}$ diazoxide 30 minutes before an i.p. injection of $0.9 \%$ saline.

5HDSAL $(n=6)$ received pretreatment with an i.p. injection of $40 \mathrm{mg} / \mathrm{kg} 5$-HD 30 minutes before an i.p. injection of $0.9 \%$ saline.

SALSAL $(n=6)$ received pretreatment with an i.p. injection of $0.9 \%$ saline 30 minutes before another i.p. injection of $0.9 \%$ saline.

SALDOX $(n=6)$ received pretreatment with an i.p. injection of $0.9 \%$ saline 30 minutes before an i.p. injection of $3 \mathrm{mg} / \mathrm{kg}$ doxorubicin.

Injections were given on day 1, 3, 5 and 7 and protocols are illustrated in Figure 1. On day 10 hearts were

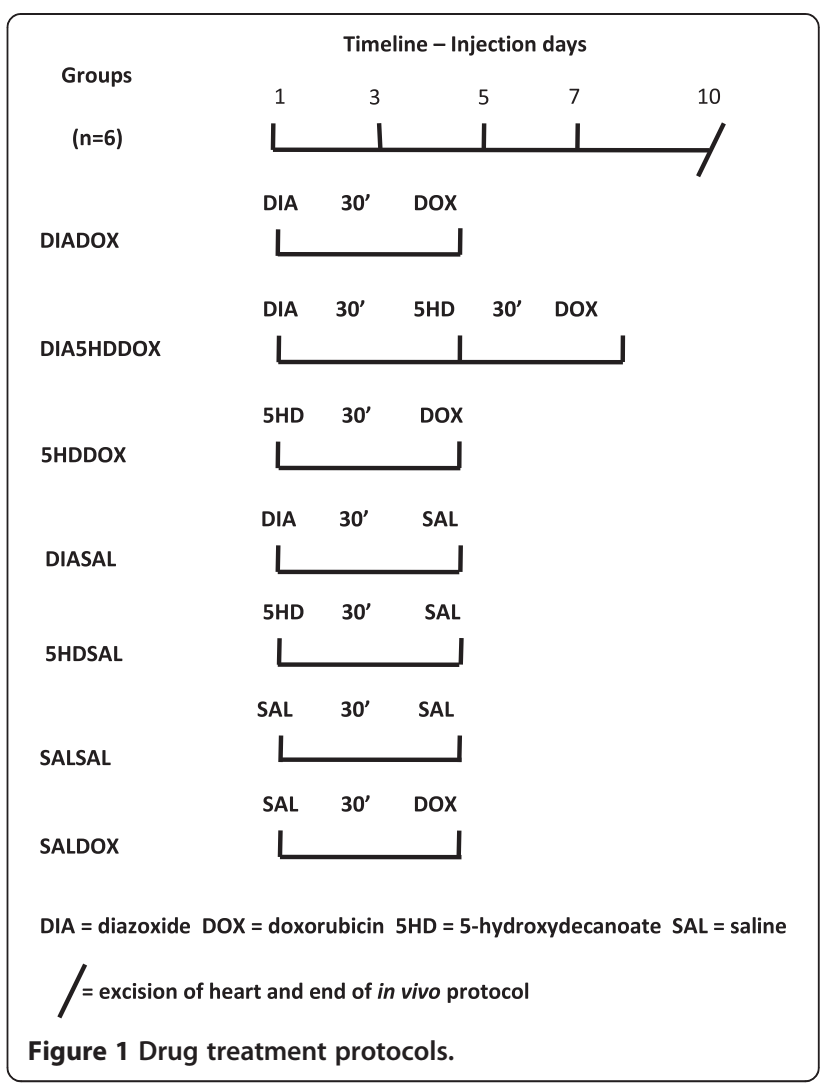

excised and Langedorff-perfused with the following protocol: 15 minutes stabilisation period with pressure-regulated flow, followed by 5 minutes with pressure-regulated flow and 5 minutes with volume-regulated flow. During the latter 10 minutes physiological data were recorded, and cardiac effluent samples collected for the evaluation of biochemical and pharmacological parameters. The perfusion protocol is illustrated in Figure 2.

\section{Perfusion protocol}

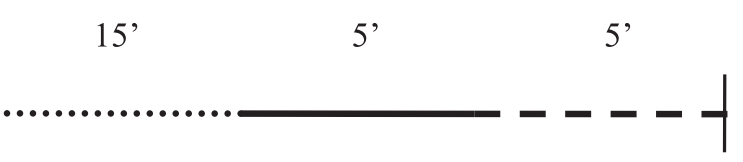

Stabilisation period

Pressure regulated perfusion

Volume regulated perfusion

End of protocol

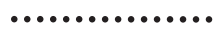

Figure 2 Perfusion protocol. 


\section{Quantification of doxorubicin and doxorubicinol}

Doxorubicin and doxorubicinol were quantified by high performance liquid chromatography (HPLC-MS/MS) (1200 series RRLC, Agilent Technologies, USA) coupled to an Agilent 6410 triple quadrupole mass spectrometer using positive electrospray ionisation (Agilent Technologies, USA). Frozen left ventricular tissue was minced and weighted out in a glass tube with a screw cap and homogenized in physiological saline $(2 \mathrm{ml} / 100 \mathrm{mg}$ tissue $)$ with a tissue homogenizer (Ultra Turrax, Sigma Aldrich, Germany). $1000 \mu \mathrm{l}$ of sample was added $100 \mu \mathrm{l}$ of daunorubicin as internal standard (IS), and $200 \mu$ lof buffer (1 M TRIZMA, $\mathrm{pH}$ 11.1) and mixed well before extraction with $4 \mathrm{ml}$ ethylacetate/heptane $(80 / 20 \mathrm{vol} / \mathrm{vol})$. The samples were mixed using a rotary blender for 15 minutes and then centrifuged at $3500 \mathrm{rpm}$ for 10 minutes at $10^{\circ} \mathrm{C}$. The organic phase was evaporated to dryness at $50^{\circ} \mathrm{C}$ under nitrogen then dissolved in $100 \mu \mathrm{l}$ of methanol followed by $100 \mu \mathrm{l}$ of distilled water. The extract was mixed thoroughly and transferred to silanized vials before analysis. $25 \mu \mathrm{l}$ of extract was injected and separated on a Zorbax SB-Aq $(2.1 \times 50 \mathrm{~mm}, 1.8 \mu \mathrm{m}$ particles, Agilent Technologies, USA) column using gradient elution with acetonitrile and $0.1 \%$ formic acid in water. Quantification was performed using multiple reaction monitoring (MRM) mode at $\mathrm{m} / \mathrm{z} 546.1 \rightarrow 363.1$ for doxorubicinol, $\mathrm{m} / \mathrm{z}$ $544.1 \rightarrow 361.1$ for doxorubicin and $\mathrm{m} / \mathrm{z} 528.1 \rightarrow 321.1$ for daunorubicin (IS).

\section{Effluent content of $\mathrm{H}_{2} \mathrm{O}_{2}$}

$\mathrm{H}_{2} \mathrm{O}_{2}$ in cardiac effluent was measured using an Apollo 4000 electrochemical detection system (World Precision Instruments, Sarasota, Florida, USA). The electrode was calibrated using 9 serial dilutions of $\mathrm{H}_{2} \mathrm{O}_{2}$ in phosphate buffered saline with added aniline. The current recorded from the effluent was then calculated as $\mu \mathrm{M} \mathrm{H} \mathrm{H}_{2} \mathrm{O}_{2}$. Samples were kept at $37^{\circ} \mathrm{C}$ during measurement. The electrode was allowed 3 minutes of stabilisation and 1 minute of recording.

\section{Effluent content of TnT}

$\mathrm{TnT}$ in cardiac effluent was measured using an Elecsys 2010 immunoassay analyzer (Roche Diagnostics Norway AS, Oslo, Norway), based on the sandwich principle. Total duration of assay: 9 minutes. 1st incubation: $50 \mu \mathrm{L}$ of sample, a biotinylated monoclonal cardiac TnTspecific antibody, and a monoclonal cardiac TnT-specific antibody labeled with a ruthenium complex (Tris(2,2bipyridyl)ruthenium(II)-complex ( $\mathrm{Ru}($ bpy))) reacted to form a sandwich complex. 2nd incubation: After addition of streptavidin-coated microparticles, the complex became bound to the solid phase via interaction of biotin and streptavidin. The reaction mixture was aspirated into the measuring cell where the microparticles were magnetically captured onto the surface of the electrode. Unbound substances were then removed with ProCell. Application of a voltage to the electrode then induced chemiluminescent emission which was measured by a photomultiplier. Results were determined via a calibration curve which was instrument-specifically generated by 2-point calibration and a master curve (5-point calibration) provided via the reagent barcode. Detection limit was $5.0 \mathrm{ng} / \mathrm{L}$.

\section{Statistics}

All results are reported as mean values \pm standard deviation (SD) in tables. Groups were compared with regards to parameters with a one-way analysis of variance (ANOVA) and subsequently Fisher's protected leastsignificant difference test. SPSS for Windows version 17.0 was used and $p<0.05$ was considered statistically significant.

\section{Results}

All physiological results from the pressure regulated perfusion are presented in Table 1 and all physiological results from the volume regulated perfusion are presented in Table 2. Biochemical results and doxorubicin analysis are presented in Table 3.

During pressure regulated perfusion LVDP was significantly $(\mathrm{p}<0.05)$ higher in DIADOX $(136.6 \pm 2.9 \mathrm{mmHg})$ compared to SALDOX $(120.4 \pm 3.6 \mathrm{mmHg})$ and LVEDP was significantly $(\mathrm{p}<0.05)$ lower in DIADOX $(9.3 \pm$ $1.5 \mathrm{mmHg})$ compared to SALDOX $(15.4 \pm 2.2 \mathrm{mmHg})$. There was no significant difference in LVDP, LVEDP or coronary flow in DIA5HDDOX or 5HDDOX versus SALDOX.

During volume regulated perfusion LVDP was significantly $(\mathrm{p}<0.05)$ higher in DIADOX $(141.5 \pm 6.9 \mathrm{mmHg})$ compared to SALDOX $(117.1 \pm 9.1 \mathrm{mmHg})$. LVEDP was significantly $(\mathrm{p}<0.05)$ lower in DIADOX $(9.6 \pm$ $2.5 \mathrm{mmHg})$ compared to SALDOX $(18.0 \pm 13.2 \mathrm{mmHg})$. AoP was significantly $(\mathrm{p}<0.05)$ lower in DIADOX $(103.9 \pm 2.2 \mathrm{mmHg})$ compared to SALDOX $(133.7 \pm$ $5.0 \mathrm{mmHg}) . \mathrm{dp} / \mathrm{dt}_{\max }$ was significantly $(\mathrm{p}<0.05)$ higher in DIADOX (3697.4 $\pm 141.0 \mathrm{mmHg} / \mathrm{s}$ ) compared to SALDOX $(2777.8 \pm 386.9 \mathrm{mmHg} / \mathrm{s}) . \mathrm{dp} / \mathrm{dt}_{\min }$ was not significantly different in DIADOX versus SALDOX, and there was no significant difference in LVDP, LVEDP, AoP, $\mathrm{dp} / \mathrm{dt}_{\max }$ or $\mathrm{dp} / \mathrm{dt}_{\text {min }}$ in DIA5HDDOX or 5HDDOX versus SALDOX. There was no significant difference in $\mathrm{dp} / \mathrm{dt}_{\max }$ or $\mathrm{dp} / \mathrm{dt}_{\text {min }}$ between any of the groups during pressure regulated perfusion.

Coronary flow was significantly higher $(\mathrm{p}<0.05)$ in DIADOX $(11.3 \pm 1.8 \mathrm{ml} / \mathrm{min})$ compared to SALDOX $(8.9 \pm 1.7 \mathrm{ml} / \mathrm{min})$ during pressure regulated perfusion. There was no significant difference in coronary flow during pressure regulated perfusion in DIA5HDDOX or 5 HDDOX versus SALDOX. 
Table 1 Physiological results from pressure regulated perfusion

\begin{tabular}{lccccccc}
\hline & DIADOX & DIA5HDDOX & 5HDDOX & DIASAL & 5HDSAL & SALSAL & SALDOX \\
\hline LVDP $(\mathrm{mmHg})$ & $136.6 \pm 2.9^{*}$ & $122.9 \pm 4.4$ & $117.5 \pm 5.2$ & $146.4 \pm 6.8^{*}$ & $144.6 \pm 5.1^{*}$ & $152.7 \pm 11.0^{*}$ & $120.4 \pm 3.6$ \\
LVEDP $(\mathrm{mmHg})$ & $9.3 \pm 1.5^{*}$ & $16.1 \pm 1.8$ & $14.9 \pm 2.7$ & $8.2 \pm 1.5^{*}$ & $8.1 \pm 1.6^{*}$ & $8.3 . \pm 1.6^{*}$ & $15.4 \pm 2.2$ \\
AoP $(\mathrm{mmHg})$ & $73.0 \pm 0$ & $73.0 \pm 0$ & $73.0 \pm 0$ & $73.0 \pm 0$ & $73.0 \pm 0$ & $73.0 \pm 0$ & $73.0 \pm 0$ \\
Heart rate (beats per minute) & $300 \pm 0$ & $300 \pm 0$ & $300 \pm 0$ & $300 \pm 0$ & $300 \pm 0$ & $300 \pm 0$ & $300 \pm 0$ \\
$\mathrm{dp} / \mathrm{dt}_{\max }(\mathrm{mmHg} / \mathrm{s})$ & $3525.5 \pm 949.9$ & $3295.3 \pm 895.9$ & $3283.4 \pm 621.9$ & $3647.4 \pm 563.7$ & $3522.5 \pm 120.1$ & $4499.1 \pm 887.2$ & $3026.6 \pm 150.0$ \\
$\mathrm{dp} / \mathrm{dt}_{\min }(\mathrm{mmHg} / \mathrm{s})$ & $-2176.5 \pm 1147.9$ & $-2012.3 \pm 282.3$ & $-1952.7 \pm 373.9$ & $-2921.9 \pm 200.3$ & $-3050.9 \pm 277.6$ & $-3060.1 \pm 311.3$ & $-2173.0 \pm 195.1$ \\
Coronary flow $(\mathrm{ml} / \mathrm{min})$ & $11.3 \pm 1.8^{*}$ & $9.5 \pm 0.7$ & $8.8 \pm 1.2$ & $11.8 \pm 1.2^{*}$ & $12.0 \pm 1.3^{*}$ & $12.6 \pm 0.4^{*}$ & $8.9 \pm 1.7$ \\
\hline
\end{tabular}

Values presented as mean \pm standard deviation (SD). *Significantly different from hearts in SALDOX, $\mathrm{p}<0.05$.

There was no significant difference in doxorubicin or doxorubicinol accumulation in myocard in any of the intervention groups versus SALDOX. TnT was significantly $(\mathrm{p}<0.05)$ lower in DIADOX $(67.3 \pm 7.9 \mathrm{ng} / \mathrm{L})$ compared to SALDOX $(121.0 \pm 17.2 \mathrm{ng} / \mathrm{L}) . \mathrm{H}_{2} \mathrm{O}_{2}$ concentration in effluate was significantly $(\mathrm{p}<0.05)$ lower in DIADOX $(54.9 \pm 2.6 \mu \mathrm{M})$ compared to SALDOX $(73.5 \pm$ 2.4 $\mu \mathrm{M})$. There was no significant difference in $\operatorname{TnT}$ or $\mathrm{H}_{2} \mathrm{O}_{2}$ in DIA5HDDOX or 5HDDOX versus SALDOX.

\section{Discussion}

Pretreatment with diazoxide attenuated doxorubicininduced cardiac dysfunction in the rat in the present study. Doxorubicin treatment produced a significant loss in left ventricular developed pressure (LVDP) $(\mathrm{p}<0.05)$ both in volume- and pressure regulated perfusion similar to previous reports $[15,16]$. Diazoxide significantly attenuated this decrease in LVDP $(\mathrm{p}<0.05)$ in both perfusion protocols. Diazoxide also improved diastolic dysfunction in doxorubicin treated hearts, by lowering the elevation of LVEDP $(\mathrm{p}<0.05)$ in both perfusion protocols, compared to animals that received both diazoxide and 5-HD. The protective effect of diazoxide is equivalent to that of ischemic preconditioning, and diazoxide is often used as a pharmacological means to induce preconditioning [7]. The drug has previously been described as an agent with a unique molecular target by opening of mitochondrial $\mathrm{K}_{\mathrm{ATP}}$-channels in cardioprotection. However, more recently a consensus seems to emerge that there are numerous effectors involved in the cardioprotective effects of diazoxide, and these effectors may synergistically contribute to its cardioprotective properties [7].

With volume regulated flow, the effects on doxorubicininduced coronary vascular resistance, can be studied in parallel with effects on myocardial contractility. During volume regulated perfusion, aortic pressure, an indirect measure of coronary resistance, was increased in hearts that received both diazoxide and 5-HD, or just 5-HD, before doxorubicin. However, diazoxide pretreatment attenuated this effect. The vasodilatory effects of diazoxide in vascular smooth muscle are due to opening of vascular $\mathrm{K}_{\mathrm{ATP}}$-channels. $\mathrm{K}_{\mathrm{ATP}}$-channels have a pronounced role in controlling coronary blood flow and the coronary reserve, particularly in the resistance arterioles [17]. In some studies diazoxide has been noted to improve coronary flow, which is associated with cardioprotection, in perfused hearts [18], despite the fact that the coronary flow reserve is low in crystalloid-perfused hearts [19]. Coronary flow was significantly higher $(\mathrm{p}<0.05)$ in DIADOX compared to SALDOX during pressure regulated perfusion. This effect was abolished with 5-HD. Based on these results, improved cardiac function associated with preserved coronary flow during pressure regulated perfusion, and attenuated increase in aortic pressure during volume regulated perfusion, could account for some of the cardioprotective effects of diazoxide.

Table 2 Physiological results from volume regulated perfusion

\begin{tabular}{|c|c|c|c|c|c|c|c|}
\hline & DIADOX & DIA5HDDOX & 5HDDOX & DIASAL & 5HDSAL & SALSAL & SALDOX \\
\hline $\operatorname{LVDP}(\mathrm{mmHg})$ & $141.5 \pm 6.9^{*}$ & $127.9 \pm 4.3$ & $108.1 \pm 9.3$ & $149.5 \pm 10.8^{*}$ & $155.6 \pm 13.0^{*}$ & $162.9 \pm 14.7^{*}$ & $117.1 \pm 9.1$ \\
\hline LVEDP (mmHg) & $9.6 \pm 2.5^{*}$ & $16.0 \pm 2.2$ & $15.8 \pm 2.1$ & $10.6 \pm 1.8^{*}$ & $8.2 \pm 1.0^{*}$ & $9.3 \pm 1.6^{*}$ & $18.0 \pm 13.2$ \\
\hline AoP $(\mathrm{mmHg})$ & $103.9 \pm 2.2^{*}$ & $124.2 \pm 5.1$ & $130.5 \pm 7.3$ & $82.7 \pm 6.0^{*}$ & $76.0 \pm 3.5^{*}$ & $76.9 \pm 3.6^{*}$ & $133.7 \pm 5.0$ \\
\hline Heart rate (beats per minute) & $300 \pm 0$ & $300 \pm 0$ & $300 \pm 0$ & $300 \pm 0$ & $300 \pm 0$ & $300 \pm 0$ & $300 \pm 0$ \\
\hline $\mathrm{dp} / \mathrm{dt}_{\max }(\mathrm{mmHg} / \mathrm{s})$ & $3697.4 \pm 141.0^{*}$ & $2512.9 \pm 302.0$ & $2950.7 \pm 237.5$ & $4583.6 \pm 546.9^{*}$ & $3935.7 \pm 213.6^{*}$ & $3973.6 \pm 246.9^{*}$ & $2777.8 \pm 386.9$ \\
\hline $\mathrm{dp} / \mathrm{dt}_{\min }(\mathrm{mmHg} / \mathrm{s})$ & $-2380.6 \pm 157.0$ & $-2176.4 \pm 154.8$ & $-2530.1 \pm 251.4$ & $-2940.8 \pm 261.6$ & $-3041.6 \pm 403.1$ & $2862.0 \pm 295.1$ & $-2579.8 \pm 329.1$ \\
\hline Coronary flow (ml/min) & $12.5 \pm 0$ & $12.5 \pm 0$ & $12.5 \pm 0$ & $12.5 \pm 0$ & $12.5 \pm 0$ & $12.5 \pm 0$ & $12.5 \pm 0$ \\
\hline
\end{tabular}

Values presented as mean \pm standard deviation (SD). *Significantly different from hearts in SALDOX, $p<0.05$. 
Table 3 Biochemical results and drug analysis

\begin{tabular}{|c|c|c|c|c|c|c|c|}
\hline & DIADOX & DIA5HDDOX & 5HDDOX & DIASAL & 5HDSAL & SALSAL & SALDOX \\
\hline Troponin -T effluate concentration ng/L & $67.3 \pm 7.9^{*}$ & $126.3 \pm 20.5$ & $130.0 \pm 16.9$ & $27.0 \pm 4.9^{*}$ & $26.8 \pm 4.9^{*}$ & $28.0 \pm 5.3^{*}$ & $121.0 \pm 17.2$ \\
\hline $\mathrm{H}_{2} \mathrm{O}_{2}$ effluate concentration $(\mu \mathrm{M})$ & $54.9 \pm 2.6^{*}$ & $72.8 \pm 9.2$ & $70.6 \pm 5.7$ & $24.8 \pm 3.0^{*}$ & $26.1 \pm 2.5^{*}$ & $22.8 \pm 1.7^{*}$ & $73.5 \pm 2.4$ \\
\hline Doxorubicin tissue concentration $\mathrm{nmol} / \mathrm{g}$ & $1.9 \pm 0.3$ & $2.7 \pm 0.4$ & $3.3 \pm 1.8$ & $0 \pm 0$ & $0 \pm 0$ & $0 \pm 0$ & $2.6 \pm 0.5$ \\
\hline Doxorubicinol tissue concentration nmol/g & $0.4 \pm 0$ & $0.7 \pm 0.3$ & $0.4 \pm 0.1$ & $0 \pm 0$ & $0 \pm 0$ & $0 \pm 0$ & $0.5 \pm 0.2$ \\
\hline
\end{tabular}

Values presented as mean \pm standard deviation (SD). *Significantly different from hearts in SALDOX, $p<0.05$.

Diazoxide pretreated hearts had lower concentrations of $\mathrm{H}_{2} \mathrm{O}_{2}$ in cardiac effluate compared to hearts that were pretreated with 5-HD and diazoxide or 5-HD alone. Diazoxide is an inhibitor of the mitochondrial complex II protein, succinate dehydrogenase (SDH). This inhibition also occurs in the heart [20]. The activity of SDH is a site of reactive oxygen species (ROS) generation, and the possibility has been raised that diazoxide and other $\mathrm{SDH}$ inhibitors mediate some of their cardioprotective effects via modulation of ROS production. Which ROS signals cardioprotection is mediated through is not fully understood [8]. Opening of mitochondrial $\mathrm{K}_{\mathrm{ATP}}$-channels leads to increased ROS, leading to a persistent open state of the channel. The ROS responsible for this is not known [7]. $\mathrm{H}_{2} \mathrm{O}_{2}$ has been proposed as one candidate for this effect, although one study concludes that $\mathrm{H}_{2} \mathrm{O}_{2}$ is not the mediator of mitochondrial $\mathrm{K}_{\mathrm{ATP}}$-channeldependent ROS signalling [8]. In our study we found higher levels of $\mathrm{H}_{2} \mathrm{O}_{2}$ in effluate from hearts that had not undergone diazoxide pretreatment before doxorubicin, or that had received both 5-HD and diazoxide before doxorubicin. The level of $\mathrm{H}_{2} \mathrm{O}_{2}$ associated with cellular signalling is much lower than the levels associated with cardiotoxicity [21]. The present study was not designed to examine signalling effects, and our observations are thus associated with the reduced cardiotoxicity of doxorubicin. This is supported by our observation of diazoxide improving diastolic dysfunction in doxorubicin treated hearts, by lowering the elevation of LVEDP. Diastolic dysfunction and contracture is proposed to be related to ROS generation [22,23].

Diazoxide pretreated hearts also had lower concentrations of TnT in cardiac effluate compared to hearts that were pretreated with 5-HD and diazoxide or 5-HD alone. Troponins are myocardial regulatory proteins, which regulate the calcium mediated actin and myosin interaction. Troponin- $\mathrm{T}$ is widely used as a specific marker to diagnose myocardial infarction. Doxorubicin is associated with increased $\mathrm{TnT}$ in serum and in heart effluate $[15,24,25]$.

Finally, pretreatment with diazoxide was not associated with decreased myocardial accumulation of doxorubicin or doxorubicinol which suggest that the protective effects did not involve a change of distribution of the anthracycline to the heart in vivo.

\section{Limitations}

In this study we wanted to investigate if opening of mitochondrial $\mathrm{K}_{\mathrm{ATP}}$-channels by diazoxide is protective against doxorubicin cardiotoxicity. Mitochondrial $\mathrm{K}_{\mathrm{ATP}}{ }^{-}$ channels have been implicated as important mediators of preconditioning [26,27]. However, the evidence for their role in preconditioning is primarily based on the effects of pharmacological agents, in particular, diazoxide and 5-HD. Diazoxide has been reported to be a specific opener of mitochondrial $\mathrm{K}_{\mathrm{ATP}}$ channels, but studies have suggested that its mechanism of cardioprotection may be due to other actions, such as inhibition of succinate dehydrogenase $[28,29]$, activation of sarcolemmal $K_{\mathrm{ATP}}$ channels [30] or transient opening of the mitochondrial permeability transition pore [31]. In addition, it might be argued that the complex metabolic effects of 5-HD severely limit its usefulness as a 'selective' blocker of mitochondrial $\mathrm{K}_{\mathrm{ATP}}$ channels [32]. Furthermore, since their identification heavily relies on the use of diazoxide as a specific opener and 5-HD as a specific blocker, the very existence of mitochondrial $K_{\text {ATP }}$ channels may be questioned. This skeptical view is supported by a study in which no changes in mitochondrial matrix volume induced by diazoxide or 5-HD could be detected [33]. Hence, one view on 5-HD is that it should no longer be considered a useful tool for studying the role of mitochondrial $\mathrm{K}_{\mathrm{ATP}}$ channels in preconditioning [32]. Thus, although diazoxide attenuated doxorubicininduced cardiac dysfunction in our results, our model was not designed to establish the precise protective mechanisms. Furthermore, we cannot exclude that the protective effects involved a change in distribution of doxorubicin to different compartments within the heart.

\section{Conclusion}

The main observation in the present results is that pretreatment with diazoxide attenuates doxorubicin-induced cardiac contractile dysfunction, and attenuates release of biomarkers of cardiotoxicity in effluate without decreasing the accumulation of doxorubicin. 5-HD completely abolished this effect of diazoxide in our rat model. A possible mechanism could be opening of mitochondrial $\mathrm{K}_{\mathrm{ATP}}$-channels. However, we cannot exclude other effects of the drug, including opening of vascular $\mathrm{K}_{\mathrm{ATP}}$-channels. The low specificity of 5-HD, calls for additional studies of mechanisms of a promising protective intervention. 


\section{Abbreviations}

5-HD: 5-hydroxydecanoate; $\mathrm{H}_{2} \mathrm{O}_{2}$ : Hydrogen peroxide; TnT: Troponin-T; LVDP: Left ventricular developed pressure; KHBB: Krebs-Henseleit bicarbonate buffer; LVEDP: Left ventricular end-diastolic pressure; AoP: Aortic pressure; $\mathrm{dp} / \mathrm{dt}_{\text {max }}$ : Left ventricular pressure first derivatives maximum; $\mathrm{dp} / \mathrm{dt}_{\min }$ : Left ventricular pressure first derivatives minimum; HPLC-MS/MS: High performance liquid chromatography; MRM: Multiple reaction monitoring; SD: Standard deviation; ANOVA: Analysis of variance; ROS: Reactive oxygen species; SDH: Succinate dehydrogenase.

\section{Competing interest}

The authors declare that they have no competing interests.

\section{Authors' contributions}

LDH and KOF carried out the pharmacological studies, and drafted the manuscript. LDH carried out the immunochemical analysis and the anima experiments. LDH and FL carried out the hydrogen peroxide analysis, and drafted the manuscript. LDH, THL and JS conceived of the study, participated in its design and coordination, performed the statistical analysis, and helped to draft the manuscript. All authors read and approved the final manuscript.

\section{Acknowledgements}

This work was supported by Bergen Heart Foundation.

\section{Author details}

${ }^{1}$ Section of Clinical Pharmacology, Laboratory of Clinical Biochemistry, Haukeland University Hospital, 5021 Bergen, Norway. ${ }^{2}$ Institute of Biomedicine, University of Bergen, 5021 Bergen, Norway. ${ }^{3}$ Department of Heart Disease, Haukeland University Hospital, 5021 Bergen, Norway. ${ }^{4}$ Institute of Clinical Science, Faculty of Medicine and Dentistry, University of Bergen, 5021 Bergen, Norway.

Received: 29 January 2014 Accepted: 21 May 2014 Published: 27 May 2014

\section{References}

1. Hiona A, Lee AS, Nagendran J, Xie X, Connolly AJ, Robbins RC, Wu JC: Pretreatment with angiotensin-converting enzyme inhibitor improves doxorubicin-induced cardiomyopathy via preservation of mitochondrial function. J Thorac Cardiovasc Surg 2011, 142(2):396-403 e393.

2. Minotti G, Menna P, Salvatorelli E, Cairo G, Gianni L: Anthracyclines: molecular advances and pharmacologic developments in antitumor activity and cardiotoxicity. Pharmacol Rev 2004, 56(2):185-229.

3. Robert J: Preclinical assessment of anthracycline cardiotoxicity in laboratory animals: predictiveness and pitfalls. Cell Biol Toxicol 2007, 23(1):27-37

4. Ferreira AL, Matsubara LS, Matsubara BB: Anthracycline-induced cardiotoxicity. CardiovasC Hematol Agents Med Chem 2008, 6(4):278-281.

5. Gan XT, Cook MA, Moffat MP, Karmazyn M: Protective effects against hydrogen peroxide-induced toxicity by activators of the ATP-sensitive potassium channel in isolated rat hearts. J Mol Cell Cardiol 1998, 30(1):33-41.

6. $\quad \mathrm{Li} W, X u B, X u J, W u$ XL: Procyanidins produce significant attenuation of doxorubicin-induced cardiotoxicity via suppression of oxidative stress. Basic Clin Pharmacol Toxicol 2009, 104(3):192-197.

7. Coetzee WA: Multiplicity of effectors of the cardioprotective agent, diazoxide. Pharmacol Therapeut 2013, 140(2):167-175.

8. Garlid AO, Jaburek M, Jacobs JP, Garlid KD: Mitochondrial reactive oxygen species: which ROS signals cardioprotection? Am J Physiol Heart Circ Physiol 2013, 305(7):H960-H968.

9. Kelishomi RB, Ejtemaeemehr S, Tavangar SM, Rahimian R, Mobarakeh Jl, Dehpour AR: Morphine is protective against doxorubicin-induced cardiotoxicity in rat. Toxicology 2008, 243(1-2):96-104.

10. McPherson BC, Yao Z: Morphine mimics preconditioning via free radical signals and mitochondrial K(ATP) channels in myocytes. Circulation 2001 103(2):290-295.

11. Barrere-Lemaire S, Combes N, Sportouch-Dukhan C, Richard S, Nargeot J, Piot C: Morphine mimics the antiapoptotic effect of preconditioning via an Ins $(1,4,5) \mathrm{P} 3$ signaling pathway in rat ventricular myocytes. Am J Physiol Heart Circ Physiol 2005, 288(1):H83-H88.
12. Ahmed LA, El-Maraghy SA: Nicorandil ameliorates mitochondrial dysfunction in doxorubicin-induced heart failure in rats: possible mechanism of cardioprotection. Biochem Pharmacol 2013, 86(9):1301-1310.

13. Abdel-Raheem IT, Taye A, Abouzied MM: Cardioprotective effects of nicorandil, a mitochondrial potassium channel opener against doxorubicin-induced cardiotoxicity in rats. Basic Clin Pharmacol Toxicol 2013, 113(3):158-166.

14. Garlid KD, Paucek P, Yarov-Yarovoy V, Murray HN, Darbenzio RB, D'Alonzo AJ, Lodge NJ, Smith MA, Grover GJ: Cardioprotective effect of diazoxide and its interaction with mitochondrial ATP-sensitive K+ channels. Possible mechanism of cardioprotection. Circ Res 1997, 81(6):1072-1082.

15. Hole LD, Larsen TH, Fossan KO, Lime F, Schjott J: A short-time model to study relevant indices of cardiotoxicity of doxorubicin in the rat. Toxicol Mech Meth 2013, 23(6):412-418.

16. Gharanei M, Hussain A, Janneh O, Maddock HL: Doxorubicin induced myocardial injury is exacerbated following ischaemic stress via opening of the mitochondrial permeability transition pore. Toxicol Appl Pharmacol 2013, 268(2):149-156.

17. Akai K, Wang Y, Sato K, Sekiguchi N, Sugimura A, Kumagai T, Komaru T, Kanatsuka H, Shirato K: Vasodilatory effect of nicorandil on coronary arterial microvessels: its dependency on vessel size and the involvement of the ATP-sensitive potassium channels. J Cardiovasc Pharmacol 1995, 26(4):541-547.

18. Feng J, Li H, Rosenkranz ER: Diazoxide protects the rabbit heart following cardioplegic ischemia. Mol Cell Biochem 2002, 233(1-2):133-138.

19. Deng $Q$, Scicli AG, Lawton C, Silverman NA: Coronary flow reserve after ischemia and reperfusion of the isolated heart. Divergent results with crystalloid versus blood perfusion. J Thorac Cardiovasc Surg 1995, 109(3):466-472

20. Hanley PJ, Daut J: K(ATP) channels and preconditioning: a re-examination of the role of mitochondrial K(ATP) channels and an overview of alternative mechanisms. J Mol Cell Cardiol 2005, 39(1):17-50.

21. Feng $\mathrm{RH}$, Zhang JC, Li XQ, Sun HL, Liu B, Zhao YZ, Tang XD, Li J: Attenuation of cardiac ischemia/reperfusion injury by transient low hydroperoxide pretreatment in rat. Zhongguo ying yong sheng li xue za zhi = Zhongguo yingyong shenglixue zazhi =Chinese J Appl Physiol 2013, 29(3):247-250.

22. Valen G, Starkopf J, Takeshima S, Kullisaar T, Vihalemm T, Kengsepp AT, Lowbeer C, Vaage J, Zilmer M: Preconditioning with hydrogen peroxide $(\mathrm{H} 2 \mathrm{O} 2)$ or ischemia in $\mathrm{H}_{2} \mathrm{O} 2$-induced cardiac dysfunction. Free Radic Res 1998, 29(3):235-245.

23. Essick EE, Wilson RM, Pimentel DR, Shimano M, Baid S, Ouchi N, Sam F: Adiponectin modulates oxidative stress-induced autophagy in cardiomyocytes. PloS one 2013, 8(7):e68697.

24. Chennuru A, Saleem MT: Antioxidant, lipid lowering, and membrane stabilization effect of sesamol against doxorubicin-induced cardiomyopathy in experimental rats. BioMed Res Int 2013, 2013:934239.

25. Fouad AA, Albuali WH, Al-Mulhim AS, Jresat I: Cardioprotective effect of cannabidiol in rats exposed to doxorubicin toxicity. Environ Toxicol Pharmacol 2013, 36(2):347-357.

26. Garlid KD, Dos Santos P, Xie ZJ, Costa AD, Paucek P: Mitochondrial potassium transport: the role of the mitochondrial ATP-sensitive $\mathrm{K}(+)$ channel in cardiac function and cardioprotection. Biochim Biophys Acta 2003, 1606(1-3):1-21.

27. Gross GJ, Peart JN: KATP channels and myocardial preconditioning: an update. Am J Physiol Heart Circ Physiol 2003, 285(3):H921-H930.

28. Lim KH, Javadov SA, Das M, Clarke SJ, Suleiman MS, Halestrap AP: The effects of ischaemic preconditioning, diazoxide and 5-hydroxydecanoate on rat heart mitochondrial volume and respiration. J Physiol 2002, 545(Pt 3):961-974.

29. Hanley PJ, Mickel M, Loffler M, Brandt U, Daut J: K(ATP) channelindependent targets of diazoxide and 5-hydroxydecanoate in the heart. J Physiol 2002, 542(Pt 3):735-741.

30. Suzuki M, Saito T, Sato T, Tamagawa M, Miki T, Seino S, Nakaya H: Cardioprotective effect of diazoxide is mediated by activation of sarcolemmal but not mitochondrial ATP-sensitive potassium channels in mice. Circulation 2003, 107(5):682-685.

31. Hausenloy D, Wynne A, Duchen M, Yellon D: Transient mitochondrial permeability transition pore opening mediates preconditioning-induced protection. Circulation 2004, 109(14):1714-1717. 
32. Hanley PJ, Drose S, Brandt U, Lareau RA, Banerjee AL, Srivastava DK, Banaszak LJ, Barycki JJ, Van Veldhoven PP, Daut J: 5-Hydroxydecanoate is metabolised in mitochondria and creates a rate-limiting bottleneck for beta-oxidation of fatty acids. J Physiol 2005, 562(Pt 2):307-318.

33. Das M, Parker JE, Halestrap AP: Matrix volume measurements challenge the existence of diazoxide/glibencamide-sensitive KATP channels in rat mitochondria. J Physiol 2003, 547(Pt 3):893-902.

doi:10.1186/2050-6511-15-28

Cite this article as: Hole et al: Diazoxide protects against doxorubicininduced cardiotoxicity in the rat. BMC Pharmacology and Toxicology 2014 15:28

\section{Submit your next manuscript to BioMed Central and take full advantage of:}

- Convenient online submission

- Thorough peer review

- No space constraints or color figure charges

- Immediate publication on acceptance

- Inclusion in PubMed, CAS, Scopus and Google Scholar

- Research which is freely available for redistribution 